\title{
Status taxonômico e distribucional do complexo Eumops bonariensis (Chiroptera: Molossidae) no sul do Brasil
}

\author{
Itiberê P. Bernardi 1, 2; João M. D. Miranda ${ }^{1} \&$ Fernando C. Passos ${ }^{1}$ \\ ${ }^{1}$ Laboratório de Biodiversidade, Conservação e Ecologia de Animais Silvestres, Departamento de Zoologia, Universidade \\ Federal do Paraná. Caixa Postal 19020, 81531-980 Curitiba, Paraná, Brasil. \\ ${ }^{2}$ Corresponding author. Rua Guerino Cerutti 161, ap. 702, Centro, 98400-000 Frederico Westphalen, Rio Grande do Sul, \\ Brasil. E-mail: sturnira@gmail.com
}

\begin{abstract}
Distibutional and taxonomic status of the Eumops bonariensis species complex (Chiroptera: Molossidae) in southern Brazil. The taxonomic status and the geographical distribution of the specimens from southern Brazil previously identified as members of the Eumops bonariensis (Peters, 1874) species complex are here reviewed based on specimens deposited in collections of the states of Paraná and Rio Grande do Sul. The results of an investigation of diagnostic morphological characters and a multivariate morphometrics analysis suggest the recognition of two forms, E. bonariensis and E. patagonicus Thomas, 1924, thus altering considerably the distributional scenario of these forms in southern Brazil.

KEY WORDS. Distribution; morphology; morphometrics; multivariate analysis.
\end{abstract}

Assim como outros gêneros de Molossidae, Eumops Miller, 1906 possui uma série de problemas taxonômicos. O número de espécies consideradas válidas varia entre oito e dez (EGER 1977, Freeman 1981, Barquez et al. 1999, Gregorin \& Taddei 2002, Hunt et al. 2003, Barquez 2006, Fabian \& Gregorin 2007). São reconhecidas oito espécies de Eumops para o Brasil (Fabian \& Gregorin 2007), enquanto para a Região Sul do Brasil são registradas seis espécies: E. perotis (Schinz, 1821), E. auripendulus (Shaw, 1800), E. glaucinus (Wagner, 1843), E. hansae Sanborn, 1932, E. bonariensis (Peters, 1874) e E. patagonicus Thomas, 1924 (Fabian \& Gregorin 2007).

Enquanto algumas espécies de Eumops são mais facilmente diferenciadas, E. bonariensis e E. patagonicus formam um complexo grupo de formas crípticas devido à sobreposição nas medidas externas e cranianas, e devido à semelhança em caracteres qualitativos (BARQuez et al. 1999, Gregorin \& TADDeI 2002, Fabian \& Gregorin 2007). Essa pronunciada semelhança é refletida no confuso e controverso histórico taxonômico destas formas.

Sanborn (1932) considerou cinco subespécies para $E$. bonariensis: E. b. bonariensis, com ocorrência para Buenos Aires na Argentina (localidade-tipo) e para Treinte y Três no Uruguai; E. b. delticus Thomas, 1923, conhecido apenas para Caldeirão na Ilha de Marajó, Brasil (localidade-tipo); E. b. nanus (Miller, 1900), conhecido apenas para Bugaba, Chiriqui no Panamá (localidade-tipo); E. b. beckeri Sanborn, 1932 ocorrendo no Paraguai, Bolívia e em Goya na Argentina e; E. b. patagonicus para Chubut na Argentina (localidade-tipo). Esse mesmo autor alertou para as semelhanças nas medidas de E. $b$. beckeri e $E$. $b$. patagonicus. Mais tarde, Cabrera (1957) considerou E. b. patagonicus como sinônimo júnior de $E$. $b$. bonariensis.
Revisando o gênero, EgER (1977), reconheceu quatro subespécies para E. bonariensis: $E$. $b$. bonariensis, no sul do Brasil até a região de Buenos Aires na Argentina; E. b. beckeri, na Bolívia, Paraguai e norte da Argentina; E. b. nanus, no sul do México, Honduras, Panamá, norte da Colômbia, Venezuela, Guiana e norte do Peru e; E. $b$. delticus na bacia Amazônica do Brasil central e Colômbia.

BARQUEZ et al. (1999) consideraram E. b. beckeri distinta de E. b. bonariensis alegando a simpatria entre estas formas nas províncias argentinas de Tucumán, Santa Fé e Buenos Aires. Dadas as semelhanças entre E. b. beckeri e o holótipo de E. patagonicus, BARQUEz et al. (1999) sinonimizaram essas duas formas sob o nome E. patagonicus já que esse tem prioridade sobre E. b. beckeri.

Hunt et al. (2003) seguiram a proposta de EgER (1977) e Koopman (1994), não considerando o status específico de $E$. patagonicus, chamando essa forma de E. b. beckeri. Dessa maneira, Hunt et al. (2003), consideraram a distribuição da forma E. b. bonariensis restrita ao sudeste de Santa Catarina, Rio Grande do Sul, Uruguai e leste da Argentina.

Simmons (2005) tratou E. bonariensis como espécie distinta de $E$. patagonicus, atribuindo três formas subespecíficas: $E$. $b$. bonariensis, $E$. $b$. delticus e $E$. $b$. nanus, enquanto considerou $E$. patagonicus sob duas formas: E. p. patagonicus e E. p. beckeri.

Nos estados da Região Sul do Brasil estas espécies possuem escassos registros e o status taxonômico destas populações não é menos complexo do que seu histórico.

No estado do Rio Grande do Sul, SiLva (1984), relatou a ocorrência de E. bonariensis não atribuindo nenhuma subespécie aos espécimes registrados. GREgorin \& TADDEI (2002) citam para o estado do Rio Grande do Sul a forma E. b. bonariensis e presumivelmente $E$. patagonicus. 
Pacheco \& Freitas (2003) listaram E. bonariensis como espécie com "dados insuficientes" no Livro Vermelho da Fauna Ameaçada de Extinção no Rio Grande do Sul, a partir dos registros disponíveis na Coleção Científica do Museu de Ciências Naturais da Fundação Zoobotânica do Rio Grande do Sul. Os autores também não atribuíram subespécie aos espécimes. De acordo com PACHeco \& Freitas (2003), E. bonariensis possui ocorrência conhecida para apenas três localidades no estado: Garruchos $\left(28^{\circ} 11^{\prime} \mathrm{S}\right.$ e $\left.55^{\circ} 38^{\prime} \mathrm{W}\right)$ no extremo noroeste, São Francisco de Paula $\left(29^{\circ} 30^{\prime} \mathrm{S}\right.$ e $\left.50^{\circ} 15^{\prime} \mathrm{W}\right)$ e Porto Alegre $\left(30^{\circ} 02^{\prime} \mathrm{S}\right.$ e $\left.51^{\circ} 13^{\prime} \mathrm{W}\right)$, ambos no leste do estado. Embora não conste nas localidades apontadas por PACHECo \& Freitas (2003), EgER (1977) apresenta cinco espécimes provenientes de Quinta $\left(32^{\circ} 05^{\prime} \mathrm{S}\right.$ e $52^{\circ} 17^{\prime} \mathrm{W}$ ) no extremo sul do Rio Grande do Sul (Fig. 1).

No mesmo ano, GonzÁlez (2003), relatou a primeira ocorrência de E. patagonicus para o Brasil baseado na re-identificação dos espécimes procedentes de Garruchos (Fig. 2). Peracchi et al. (2006) aceitaram E. patagonicus como espécie válida e consideraram E. bonariensis uma espécie amplamente distribuída pela região Neotropical, não atribuindo categoria subespecífica aos registros. Fabian \& GRegorin (2007) consideram a ocorrência de $E$. bonariensis no Rio Grande do Sul, mas estenderam a distribuição de E. patagonicus até as localidades de ocorrência de E. bonariensis do leste do estado do Rio Grande do Sul (Fig. 3).

No estado do Paraná, Miretzki (2003) relata a presença de E. bonariensis a partir de sete espécimes depositados na coleção do Museu de História Natural do Capão da Imbuia, Curitiba. Os espécimes são procedentes de três localidades: Douradina $\left(23^{\circ} 22^{\prime} \mathrm{S}\right.$ e $\left.053^{\circ} 17^{\prime} \mathrm{W}\right)$ no noroeste do estado, União da Vitória $\left(26^{\circ} 13^{\prime} \mathrm{S}\right.$ e $\left.051^{\circ} 05^{\prime} \mathrm{W}\right)$ no sul e Curitiba $\left(25^{\circ} 28^{\prime} \mathrm{S}\right.$ e $\left.049^{\circ} 17^{\prime} \mathrm{W}\right)$ no leste (Figs 1-3). O autor não atribuiu subespécie aos espécimes.

O presente trabalho tem por objetivo revisar a identidade específica e a distribuição geográfica dos espécimes sul-brasileiros atribuídos a E. bonariensis e E. patagonicus.

\section{MATERIAL E MÉTODOS}

Foram examinados os espécimes atribuídos a E. bonariensis e E. patagonicus citados na literatura para o sul do Brasil (Gregorin \& Taddei 2002, González 2003, Miretzki 2003, Pacheco \& Freitas 2003, Peracchi et al. 2006, Fábian \& Gregorin 2007), além de espécimes adicionais disponíveis nas seguintes coleções mastozoológicas: Museu de Ciências Naturais, da Fundação Zoobotânica do Rio Grande do Sul, Porto Alegre (MCN), Museu de História Natural do Capão da Imbuia, Curitiba (MHNCI) e Coleção Científica de Mastozoologia do Departamento de Zoologia, Universidade Federal do Paraná, Curitiba (DZUP/CCMZ). Dois espécimes citados por Miretzki (2003), um procedente de Douradina (Paraná) e outro de Curitiba (Paraná) não foram localizados na coleção do MHNCI.

Com exceção dos 11 espécimes atribuídos à E. patagonicus por GonzÁlez (2003) todos os demais espécimes utilizados no presente trabalho tiveram os crânios retirados e preparados pelo primeiro autor (IPB).
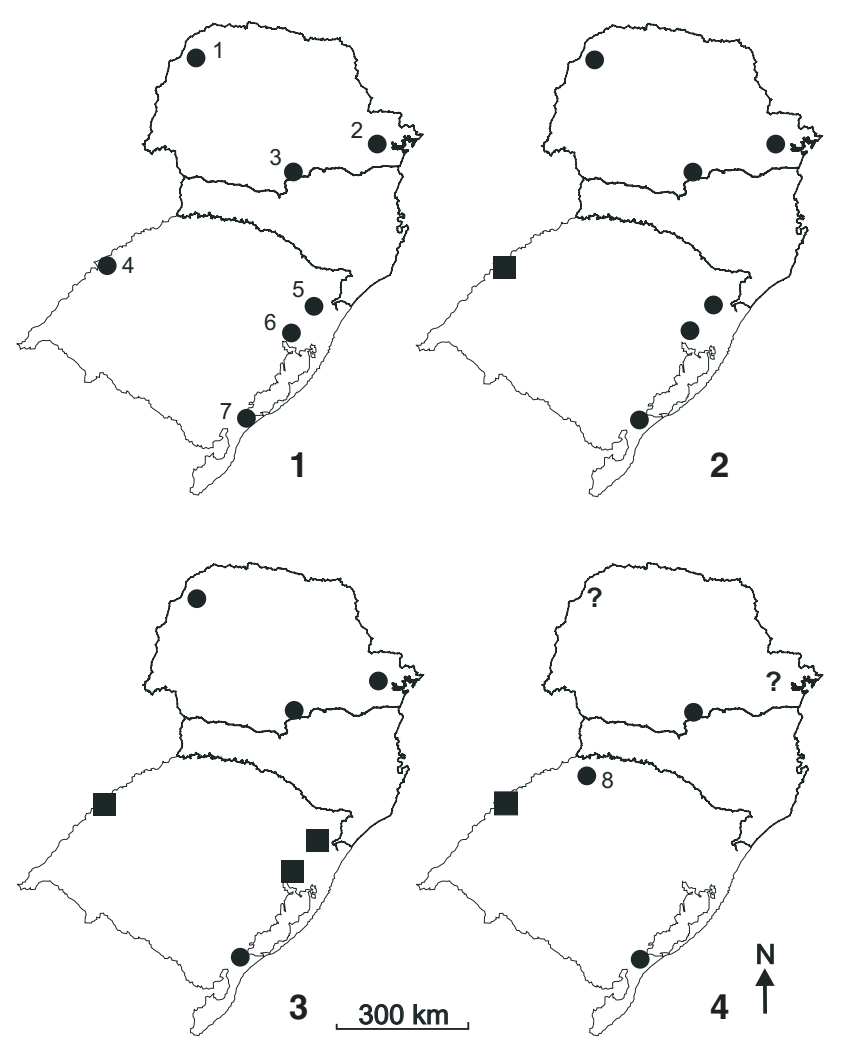

Figuras 1-4. Histórico distribucional de E. bonariensis (círculos) and E. patagonicus (quadrados) no sul do Brasil: (1) distribuição baseada em Eger (1977), Pacheco \& Freitas (2003) e Miretzki (2003); (2) Alteração apresentada em GonZÁlez (2003); (3) alteração apresentada em Fabí́n \& GreGorin (2007); (4) presente estudo. (1) Douradina; (2) Curitiba; (3) União da Vitória; (4) Garruchos; (5) São Francisco de Paula; (6) Porto Alegre; (7) Quinta; (8) Frederico Westphalen.

Foram analisados os caracteres qualitativos diagnósticos para a diferenciação das formas de acordo com SANBORN (1932), Eger (1977) Barquez et al. (1999) e Gregorin \& TAddei (2002) a constar: alcance da quilha interna da orelha, formato do trago, lâmina de separação entre as fossas basisfenóides e forma do crânio.

Com auxílio de um paquímetro com aproximação para 0,05 $\mathrm{mm}$ foram aferidas as seguintes métricas: comprimento do antebraço (An) - maior comprimento obtido no antebraço incluindo o carpo; comprimento total do crânio (incluindo incisivos) (CCRi) - da região mais posterior do occipital à frente dos incisivos centrais; comprimento total do crânio (excluindo incisivos) (CCR) - da região mais posterior do occipital à região proximal dos incisivos centrais; comprimento côndiloincisivo (Ci) - do centro da linha de conexão entre os côndilos occipitais à frente dos incisivos centrais; comprimento côndilobasal $(\mathrm{Cb})$ - do centro da linha de conexão entre os côndilos occipitais até a região proximal dos incisivos centrais; compri- 
mento côndilo-canino (Cc) - da face posterior dos côndilos occipitais à frente do canino do lado correspondente; comprimento da série de dentes superiores $(\mathrm{C}-\mathrm{M})$ - da região mais posterior do último molar à face anterior do canino do lado correspondente; comprimento da série de dentes inferiores (cm) - da região mais posterior do último molar à face anterior do canino do lado correspondente (na mandíbula); comprimento da mandíbula $(\mathrm{Cm})$ - da região anterior dos incisivos centrais à linha de conexão dos processos condilares; largura da constrição pós-orbitária (Lpo) - a menor distância obtida na constrição pós-orbitária; largura zigomática (Lz) - a maior distância transversal obtida ao longo dos arcos zigomáticos; largura através dos caninos (C-C) - maior distância obtida através dos "cingula" dos caninos; largura através dos molares (M-M) - a maior distância obtida através das bordas vestibulares dos molares; largura da caixa craniana (Lcx) - a maior distância obtida na caixa craniana, imediatamente acima da região escamosal e; largura mastóidea (Lmt) - a maior distância obtida através dos processos mastóideos.

Como análise exploratória as variáveis cranianas originais foram submetidas a uma Análise de Componentes Principais (ACP), a partir de uma matriz de correlações. As relações fenéticas entre as localidades foram testadas utilizando as variáveis originais de cada indivíduo em uma Análise de Agrupamento (Cluster) por similaridade Euclidiana. Ambas as análises foram realizadas no software Past versão 1.81 (disponível em: http://folk.uio.no/ohammer/past). Como análise confirmatória os escores dos três primeiros componentes foram submetidos a uma Análise Canônica Discriminante no pacote JMP versão 5.0.1.

Os mapas com as distribuições geográficas foram construídos com base nos dados obtidos diretamente das etiquetas dos espécimes e/ou dos livros tombo de sua respectiva coleção.

\section{RESULTADOS E DISCUSSÃO}

\section{Material examinado}

Foram examinados 18 espécimes do Rio Grande do Sul: 14 procedentes de Garruchos (MCN 356-369), um de Porto Alegre (MCN 1027), dois de São Francisco de Paula (MCN 822 e 823) e um de Frederico Westphalen (DZUP/CCMZ 526); e cinco espécimes procedentes de União da Vitória no estado do Paraná (MHNCI 2840, 2842, 2858, 2863 e 2864).

Espécimes examinados: Eumops patagonicus $(\mathrm{n}=14)$ MCN 00356-00369, Garruchos, Rio Grande do Sul, Brasil $\left(28^{\circ} 11^{\prime} \mathrm{S}\right.$ e $\left.55^{\circ} 38^{\prime} \mathrm{W}\right)$. Eumops bonariensis $(\mathrm{n}=5)$ - MHNCI 2840, 2842, 2858, 2863 e 2864. União da Vitória, Paraná, Brasil $\left(26^{\circ} 13^{\prime}\right.$ S e $\left.51^{\circ} 05^{\prime} \mathrm{W}\right)$; $(\mathrm{n}=1)$ - DZUP/CCMZ 526, Frederico Westphalen, Rio Grande do Sul, Brasil $\left(27^{\circ} 21^{\prime}\right.$ S e $\left.53^{\circ} 23^{\prime} \mathrm{W}\right)$. Molossus sp. ( $\mathrm{n}=2)$ - MCN 822, 823, São Francisco de Paula, Rio Grande do Sul, Brasil. (29 $30^{\prime} \mathrm{S}$ e $\left.50^{\circ} 15^{\prime} \mathrm{W}\right)$. Tadarida brasiliensis $(\mathrm{n}=1)-\mathrm{MCN} 1027$, Porto Alegre, Rio Grande do Sul, Brasil. $\left(30^{\circ} 02^{\prime} \mathrm{S}\right.$ e $\left.51^{\circ} 13^{\prime} \mathrm{W}\right)$.
Os espécimes procedentes de São Francisco de Paula (MCN 822 e 823) apresentaram o seguinte conjunto de caracteres: (1) quilha na região mediana do focinho, (2) antitrago bem desenvolvido, arredondado e com uma acentuada constrição na base, (3) crânio com crista sagital bem desenvolvida na região anterior e (4) palato raso, não em domo. Esse conjunto de caracteres permitiu identificar esses espécimes como morcegos Molossus E. Geoffroy (Dolan 1989, Barquez et al. 1999, Gregorin \& Taddei 2002). O espécime MCN 1027 procedente de Porto Alegre consiste apenas de um crânio, sem a mandíbula e sem a maioria dos dentes. As poucas dimensões mensuráveis, o formato do rostro, a reentrância palatal e a presença de um dos incisivos permitiram identificar o material como pertencente à Tadarida brasiliensis (I. Geoffroy, 1824) (BARQuEz et al. 1999, GREgorin \& TADDEI 2002). Assim, esses espécimes (MCN 822, 823 e 1027) não constarão nas demais análises.

\section{Morfologia}

Quilha interna da orelha e trago: os 11 espécimes procedentes de Garruchos e atribuídos a E. patagonicus por GonZÁLEZ (2003), não puderam ser avaliados, já que estão preparados em via seca e o ressecamento das membranas deformou estas estruturas. Os espécimes MCN 367 e 369, conservados em via úmida, apresentaram a quilha interna da orelha não ultrapassando a borda anterior do antitrago como descrito para $E$. patagonicus (ver BARQUEZ et al. 1999: 194, fig. 138), embora no espécime MCN 368 a quilha alcance o meio do antitrago como apresentado por Eger (1977: 33, fig. 18), para E. b. beckeri. O trago destes espécimes também se assemelha ao apresentado por BARQUEZ et al. (1999) para E. patagonicus.

No espécime de Frederico Westphalen (DZUP/CCMZ 526) a quilha interna da orelha alcança a borda posterior do antitrago e o trago assemelha-se ao citado por BARQuez et al. (1999) para E. b. bonariensis.

Os espécimes de União da Vitória (MHNCI 2840, 2842, 2858, 2863 e 2864) apresentam para estes caracteres estados intermediários entre E. patagonicus e E. b. bonariensis (sensu BARQUEz et al. 1999), entretanto essa variação pode ser algum artifício de preparação, tornando difícil sua avaliação. Desta forma, estes caracteres podem ser utilizados como ferramentas auxiliares, mas de forma isolada tornam-se pouco conclusivos.

Fossas basisfenóides: nos 14 espécimes de Garruchos a lâmina que separa as fossas basisfenóides apresentou grande variação, desde extremamente estreita e delgada até mais larga e robusta (Figs 5 e 6). No espécime de Frederico Westphalen as fossas basisfenóides são separadas por uma larga lâmina óssea (Fig. 8). Dois dos espécimes de União da Vitória (MHNCI 2842 e 2864) apresentaram a lâmina tão larga e robusta (Fig. 7) quanto o espécime de Frederico Westphalen. Já os espécimes MHNCI 863 e 2840 apresentaram lâminas de espessura intermediária entre os espécimes de Garruchos e o de Frederico Westphalen, enquanto o espécime MHNCI 2858 apresentou a lamina extremamente estreita e delgada. 

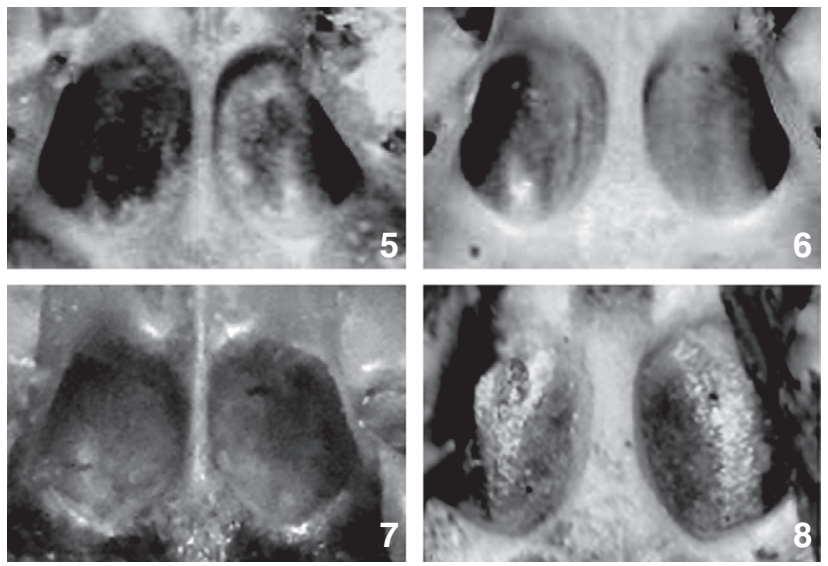

Figuras 5-8. Extremos da variação na espessura das lâminas de separação das fossas basisfenóides nos espécimes examinados: (5) MCN 358, Garruchos; (6) MCN 360, Garruchos; (7) MHNCI 2858, União da Vitória; (8) DZUP 526, Frederico Westphalen.

Forma do crânio: os espécimes de Garruchos apresentaram a região anterior do basicrânio mais inflada, conferindo um aspecto mais globoso como descrito para E. patagonicus (BARQUeZ et al. 1999, GREgorin \& TADDEI 2002). O crânio do espécime de Frederico Westphalen não apresenta a região anterior do basicrânio inflada, o que lhe confere um aspecto mais alongado, como descrito para E. b. bonariensis (BARQUEZ et al. 1999, Gregorin \& TADDei 2002). Quatro dos espécimes de União da Vitória apresentaram formas intermediárias, variando desde mais globosos (e.g. MHNCI 2864) até mais alongados (e.g. MNHCI 2842). Dada a variação encontrada consideramos este caractere também pouco conclusivo.

\section{Morfometria}

As métricas aferidas nos espécimes examinados são apresentadas na tabela I. Para os espécimes de Garruchos em via seca (MCN 356-366) assumimos as medidas de antebraço descritas por GonZález (2003) para efeitos de comparação com as tabelas II e III.

Os espécimes de Garruchos apresentaram as menores medidas, como em E. patagonicus (BARQUEZ et al. 1999, GREGorin $\&$ TADDEI 2002). O espécime de Frederico Westphalen apresentou dimensões, semelhantes às apresentadas por BARQUEZ et al. (1999) para E. b. bonariensis. Os espécimes de União da Vitória apresentaram o comprimento do antebraço, contemplando grande parte da variação encontrada no complexo E. bonariensis (SANBorn 1932, Eger 1977, BARquez et al. 1999, GREgorin \& TADDEI 2002, Hunt et al. 2003) e apresentaram também as maiores dimensões cranianas. O espécime MHNCI 2842 apresentou o maior comprimento de antebraço e as menores dimensões cranianas (Tab. I).

Tabela I. Medidas ( $\mathrm{mm}$ ) obtidas nos espécimes examinados. Ver acrônimos e descrição das medidas no texto.

\begin{tabular}{|c|c|c|c|c|c|c|c|c|c|c|c|c|c|c|c|}
\hline Número tombo/sexo & An & $\mathrm{CCR}(\mathrm{i})$ & $\mathrm{CCR}$ & $\mathrm{Cb}$ & $\mathrm{Ci}$ & $\mathrm{Cc}$ & Lpo & Lz & $\mathrm{Lmt}$ & Lcx & $\mathrm{C}-\mathrm{C}$ & M-M & C-M & $\mathrm{Cm}$ & $\mathrm{c}-\mathrm{m}$ \\
\hline MCN 356/F & 44,1 & & & & & 16,3 & 4,4 & 11,1 & 9,5 & 9,1 & 4,5 & 8,1 & 7,0 & & 7,3 \\
\hline MCN 357/F & 44,6 & & & & & 16,5 & 4,3 & 11,0 & 10,0 & 9,0 & 4,4 & 8,0 & 7,1 & & \\
\hline MCN 358/F & 44,6 & & & 16,1 & 17,1 & 16,1 & 4,1 & & 10,2 & 9,1 & & 7,9 & 7,1 & & 7,5 \\
\hline MCN 359/F & 45,1 & 18,9 & 18,1 & 16,9 & 17,3 & 16,2 & 4,3 & 11,2 & 10,5 & 9,2 & 4,3 & 7,9 & 6,9 & 12,3 & 7,2 \\
\hline MCN 360/M & 44,0 & 18,8 & 17,8 & 16,0 & 17,1 & 16,2 & 4,3 & 11,2 & 10,3 & 9,3 & 4,3 & 8,3 & 7,2 & & 7,6 \\
\hline MCN 361/M & 44,7 & 17,5 & 16,7 & 16,0 & 16,6 & 15,7 & 4,1 & & 9,5 & 8,8 & 4,5 & 7,6 & 7,0 & 11,9 & 7,4 \\
\hline MCN 362/F & 42,0 & 17,8 & 17,1 & 16,2 & 16,7 & 15,8 & 4,1 & 10,9 & 9,8 & 8,9 & 4,4 & 7,8 & 6,7 & & 7,1 \\
\hline MCN 363/M & 45,5 & 18,1 & 17,9 & 16,9 & 17,0 & 16,4 & 4,1 & 11,0 & 10,3 & 9,0 & 4,6 & 8,2 & 7,3 & 12,2 & 7,5 \\
\hline MCN 364/F & 45,7 & & & & & 16,0 & 4,1 & 11,0 & 9,9 & 8,9 & 4,3 & 7,6 & 6,7 & & 7,1 \\
\hline MCN 365/F & 43,9 & 18,2 & 17,6 & 16,6 & 16,8 & 16,0 & 4,3 & 10,7 & 10,3 & 9,0 & 4,5 & 7,8 & 7,1 & & 7,3 \\
\hline MCN 366/F & 43,0 & 17,8 & 16,8 & 16,5 & 17,3 & 16,5 & 4,2 & & 9,8 & 8,7 & 4,5 & 7,8 & 6,9 & & 7,3 \\
\hline $\mathrm{MCN} 367 / \mathrm{F}$ & 45,1 & 18,3 & 17,4 & 16,2 & 16,8 & 15,9 & 4,2 & 11,1 & 10,1 & 9,3 & 4,4 & 8,2 & 6,9 & 11,6 & 7,4 \\
\hline MCN 368/F & 45,5 & 18,5 & 17,9 & 16,7 & 17,3 & 16,3 & 4,5 & 11,2 & 10,6 & 9,5 & 4,4 & 7,9 & 7,1 & & 7,4 \\
\hline MCN 369/F & 45,0 & 18,2 & 17,4 & 16,2 & 16,6 & 15,9 & 4,5 & 11,2 & 10,1 & 9,5 & 4,4 & 7,9 & 6,9 & 11,8 & 7,4 \\
\hline DZUP/CCMZ 526/M & 47,7 & 19,4 & 18,5 & 17,5 & 18,2 & 17,3 & 4,1 & 11,0 & 10,5 & 9,0 & 4,6 & 8,0 & 7,2 & 12,9 & 7,5 \\
\hline $\mathrm{MHNCl} 2840 / \mathrm{M}$ & 46,6 & 19,9 & 19,0 & 18,0 & 18,5 & 17,6 & 4,2 & 11,2 & 10,6 & 9,2 & 4,7 & 8,2 & 7,4 & 13,1 & 7,8 \\
\hline $\mathrm{MHNCl} 2842 / \mathrm{F}$ & 47,6 & 19,2 & 18,5 & 17,8 & 18,4 & 17,7 & 4,2 & 11,1 & 10,5 & 9,1 & 4,6 & 8,1 & 7,3 & 13,0 & 7,7 \\
\hline $\mathrm{MHNCI} 2858 / \mathrm{M}$ & 45,5 & 19,3 & 18,6 & 17,9 & 18,1 & 17,4 & 4,0 & 10,9 & 10,2 & 9,0 & 4,4 & 8,0 & 7,6 & 12,9 & 7,8 \\
\hline $\mathrm{MHNCl} 2863 / \mathrm{M}$ & 46,5 & 19,5 & 18,8 & 17,8 & 18,3 & 17,6 & 4,2 & 11,1 & 10,7 & 9,1 & 4,4 & 8 & 7,3 & 3,1 & 7,7 \\
\hline $\mathrm{MHNCl} 2864 / \mathrm{F}$ & 45,9 & 19,4 & 18,6 & 17,6 & 18 & 17,3 & 4 & 11,4 & 10,6 & 9,2 & 4,6 & 8,1 & 7,2 & 12,8 & 7,7 \\
\hline
\end{tabular}


Tabela II. Medidas (mm) de E. patagonicus obtidos da literatura: 1) Argen-tina (BArQuez et al. 1999); 2) Oeste do Paraguai, 3) Leste do Paraguai (Célia López-González, com. pess.). Descrições das medidas constam no texto.

\begin{tabular}{|c|c|c|c|c|c|}
\hline \multirow{2}{*}{ Medidas } & \multirow{2}{*}{$\frac{1}{M+F}$} & \multicolumn{2}{|c|}{2} & \multicolumn{2}{|c|}{3} \\
\hline & & M & $\mathrm{F}$ & M & $\mathrm{F}$ \\
\hline An & $44,6(42,4-47,0)$ & $43,2(42,0-44,8)$ & $43,8(42,2-45,9)$ & $43,5(41,3-45,0)$ & $44,0(40,0-47,0)$ \\
\hline $\mathrm{CCR}$ & $17,3(16,0-18,1)$ & $17,9(17,4-18,5)$ & $17,8(16,8-18,6)$ & $18,4(17,6-18,9)$ & $18,1(17,4-18,9)$ \\
\hline $\mathrm{Cb}$ & $16,3(15,4-16,9)$ & - & - & - & - \\
\hline $\mathrm{Ci}$ & - & $16,7(16,3-17,4)$ & $16,6(15,5-17,3)$ & $17,2(16,4-17,7)$ & $16,9(16,2-18,3)$ \\
\hline Lpo & $4,1(3,9-4,5)$ & $4,2(4,0-4,3)$ & $4,1(3,8-4,5)$ & $4,2(4,0-4,4)$ & $4,1(3,9-4,4)$ \\
\hline Lz & $10,8(10,4-11,2)$ & $10,7(10,5-11,2)$ & $10,7(10,1-11,2)$ & $11,1(10,6-11,5)$ & $10,8(10,2-11,3)$ \\
\hline $\mathrm{Lmt}$ & $10,2(9,6-10,7)$ & $10,1(9,8-10,4)$ & $10,1(9,6-10,7)$ & $10,3(9,8-10,7)$ & $10,2(9,7-10,8)$ \\
\hline Lcx & $8,9(8,6-9,3)$ & - & - & - & - \\
\hline $\mathrm{C}-\mathrm{C}$ & 4,3 & $4,1(3,9-4,4)$ & $4,1(3,7-4,3)$ & $4,4(3,9-4,6)$ & $4,2(3,9-4,5)$ \\
\hline M-M & 7,8 & $7,6(7,0-7,9)$ & $7,5(7,0-7,9)$ & $7,7(7,3-8,2)$ & $7,6(7,0-8,0)$ \\
\hline C-M & $6,7(6,5-7,0)$ & $6,5(6,4-6,7)$ & $6,4(6,1-6,8)$ & $6,6(6,0-7,0)$ & $6,5(6,1-6,9)$ \\
\hline $\mathrm{Cm}$ & $12,2(11,8-12,6)$ & $12,6(12,3-13,1)$ & $12,4(11,5-13,1)$ & $13,1(12,5-13,5)$ & $12,6(11,9-13,4)$ \\
\hline $\mathrm{c}-\mathrm{m}$ & $7,3(6,9-7,7)$ & $7,1(6,9-7,3)$ & $6,9(6,3-7,2)$ & $7,3(7,0-7,8)$ & $7,0(6,7-7,4)$ \\
\hline
\end{tabular}

Tabela III. Medidas (mm) de E. bonariensis obtidos da literatura: 1) Argentina (BARQuEz et al. 1999); 2) Paraguai (Célia López-González, com. pess.); 3) Argentina (Mares et al. 1996); 4) Uruguai (Acosta Y LARA 1950). Descrições das medidas constam no texto.

\begin{tabular}{|c|c|c|c|c|c|c|c|}
\hline \multirow{2}{*}{ Medidas } & \multirow{2}{*}{$\begin{array}{c}1 \\
M+F\end{array}$} & \multicolumn{2}{|r|}{2} & \multicolumn{2}{|c|}{3} & \multicolumn{2}{|c|}{4} \\
\hline & & M & $\mathrm{F}$ & M & $\mathrm{F}$ & M & $\mathrm{F}$ \\
\hline An & $47,8(46,0-49,1)$ & $46,1(45,0-47,2)$ & $45,4(45,1-45,9)$ & $47,9(47,3-48,7)$ & $48,0(44,8-49,0)$ & 50,0 & $48,3-48,9$ \\
\hline $\mathrm{CCR}$ & $19,1(18,7-19,7)$ & $19,5(19,2-19,7)$ & $19,3(18,8-19,7)$ & $19,3(19,1-19,5)$ & $18,9(18,3-19,2)$ & 19,3 & $19,2-19,7$ \\
\hline $\mathrm{Cb}$ & $18,2(17,5-18,8)$ & $18,4(18,3-18,5)$ & $18,1(17,6-18,6)$ & $18,3(18,1-18,6)$ & $18,0(17,2-18,4)$ & $18,0-18,8$ & $18,2-19,0$ \\
\hline $\mathrm{Ci}$ & - & - & - & - & - & - & - \\
\hline Lpo & $4,2(4,0-4,5)$ & - & - & - & - & 4,0 & $4,0-4,1$ \\
\hline Lz & $11,7(11,1-12,3)$ & $11,3(11,2-11,5)$ & $11,2(11,2-11,3)$ & $11,8(11,5-12,0)$ & $11,5(11,1-11,7)$ & 12,0 & $11,5-12,0$ \\
\hline Lmt & $11,0(10,7-11,6)$ & $10,7(10,4-10,9)$ & $10,5(10,3-10,8)$ & - & - & - & - \\
\hline LCx & $9,4(8,8-10,0)$ & - & - & $9,6(9,5-9,7)$ & $9,3(8,8-9,7)$ & $9,0-9,6$ & $9,5-9,6$ \\
\hline $\mathrm{C}-\mathrm{C}$ & $4,9(4,7-5,2)$ & $4,5(4,3-4,6)$ & $4,6(4,4-4,7)$ & - & - & $4,6-4,9$ & $4,9-5,0$ \\
\hline M-M & $8,3(8,1-8,7)$ & $8,0(7,8-8,1)$ & $7,9(7,8-8,0)$ & - & - & - & - \\
\hline C-M & $7,4(7,3-7,7)$ & $7,2(7,1-7,5)$ & $6,8(6,8-6,9)$ & $7,5(7,3-7,6)$ & $7,3(7,1-7,5)$ & 7,2 & 7,1 \\
\hline $\mathrm{Cm}$ & $13,7(13,4-14,2)$ & - & - & $14,0(13,8-14,1)$ & $13,8(13,6-14,0)$ & - & - \\
\hline $\mathrm{c}-\mathrm{m}$ & $7,9(7,7-8,4)$ & $7,7(7,4-8,0)$ & $7,4(7,4-7,5)$ & $6,7(6,6-6,9)$ & $6,7(6,5-7,3)$ & 8,0 & $8,0-8,1$ \\
\hline
\end{tabular}

Na Análise de Componentes Principais o CP1 explica 47,6\% do total de variação na mostra, enquanto o CP2 e o CP3 explicam $27 \%$ e 9,7\% respectivamente. Essa análise sugere o reconhecimento de duas formas (Figs 9 e 10), sendo a primeira composta pelos espécimes procedentes de União da Vitória juntamente com o espécime procedente de Frederico Westphalen e, a segunda, composta apenas pelos espécimes procedentes de Garruchos.
Na análise de agrupamento o espécime de Frederico Westphalen foi agrupado com os espécimes de União da Vitória enquanto os espécimes de Garruchos formaram um grupo distinto (Fig.11) como sugerido pela ACP.

A Análise Canônica Discriminante confirmou os agrupamentos sugeridos pela ACP e pela análise de agrupamento, com a alocação correta de 100\% dos espécimes (Fig. 12). 

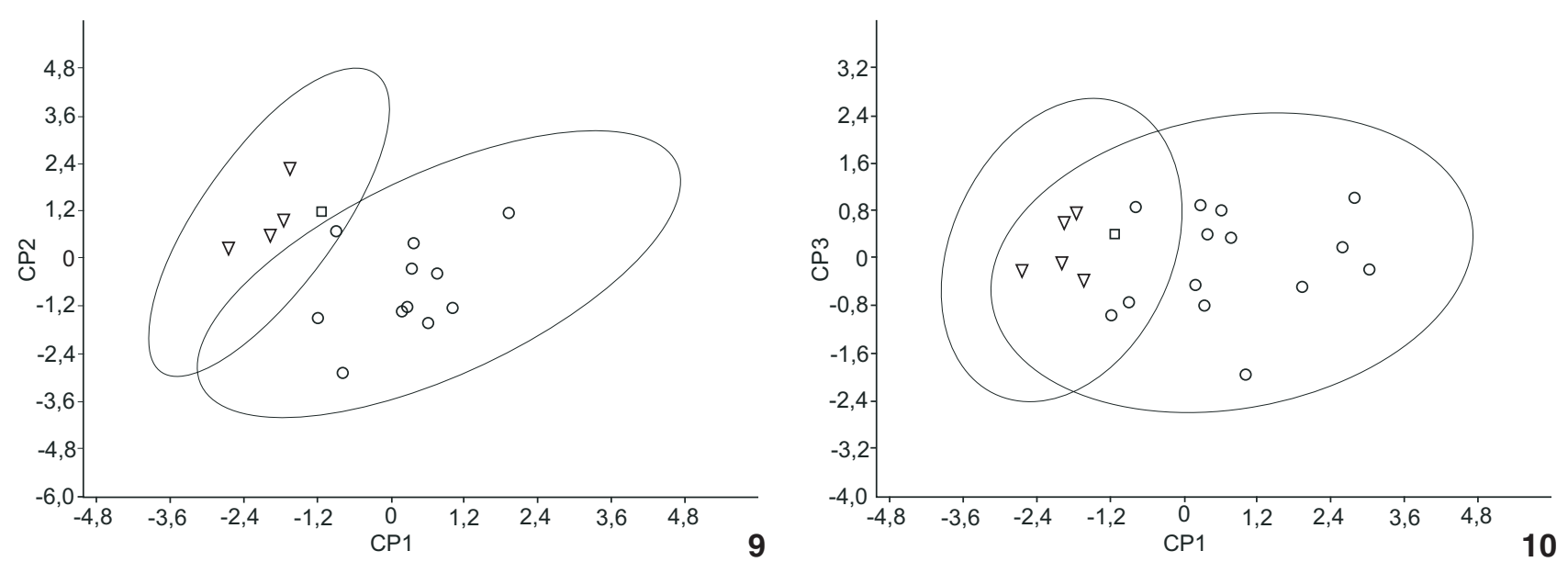

Figuras 9-10. Representação gráfica da Análise de Componentes Principais - (9) CP1 x CP2 e (10) CP1 x CP3) - gerado a partir da análise craniométrica de Eumops bonariensis de Frederico Westphalen (quadrado), União da Vitória (triângulo) e de E. patagonicus de Garruchos (círculos). Elipses de $95 \%$ de confiança.

Frederico Westphalen, RS

União da vitória, $\mathrm{PR}$

Garruchos, RS

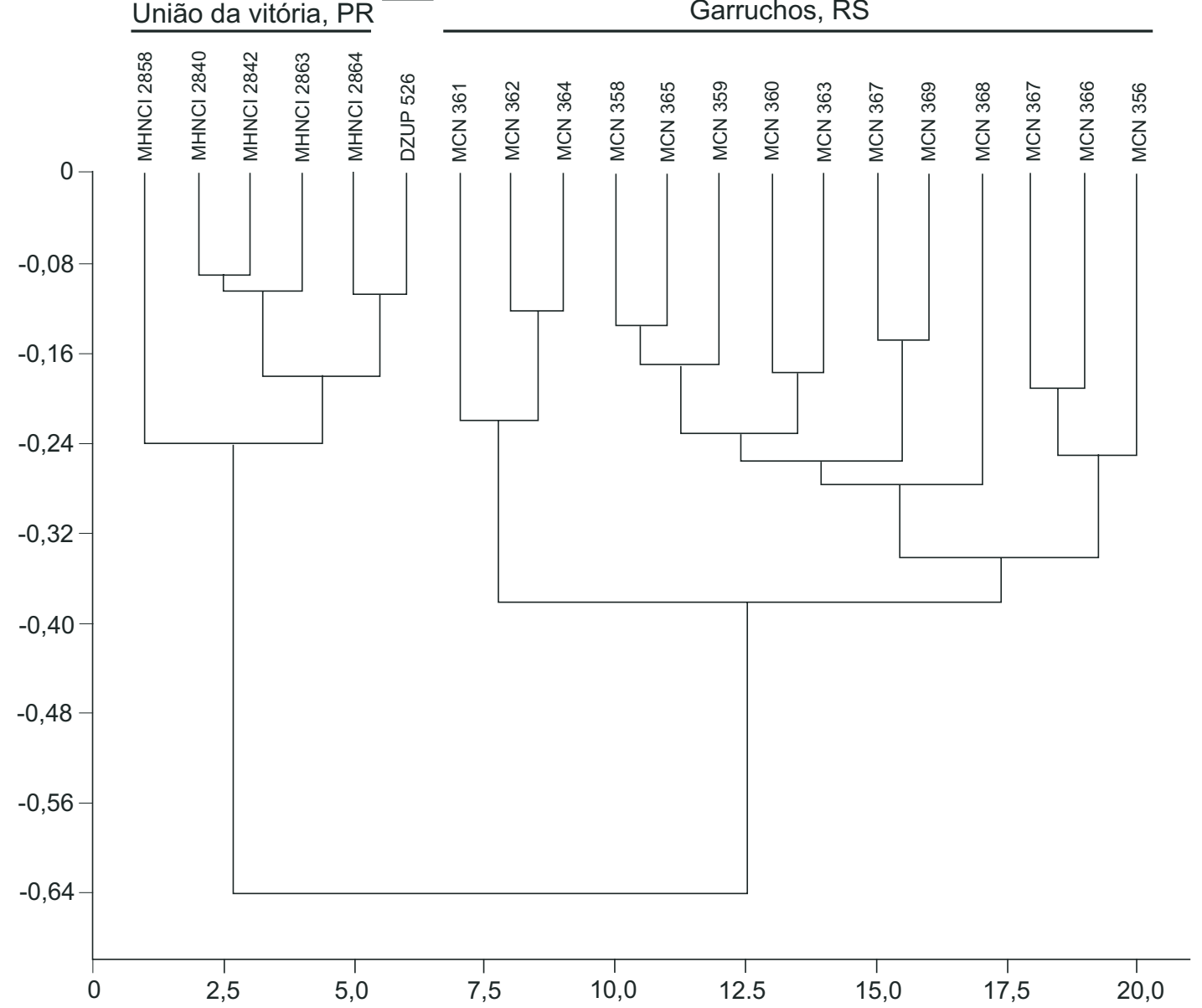

Figura 11. Análise de agrupamento por similaridade Euclidiana demonstrando as relações fenéticas entre os espécimes examinados. 


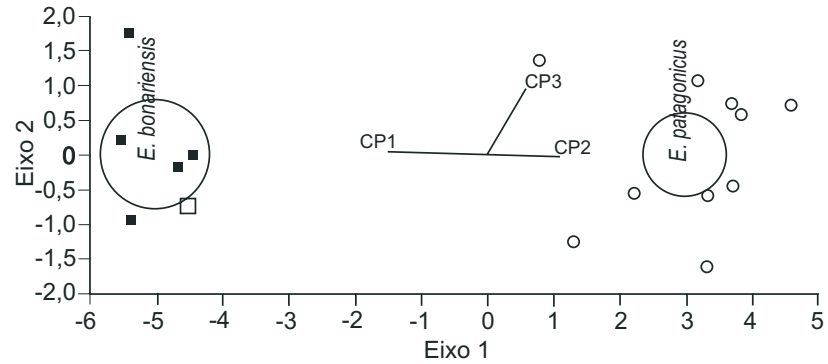

Figura 12. Representação gráfica da Análise Canônica Discriminante utilizando os três primeiros componentes principais (PC1, PC2 e PC3) para os espécimes de Eumops bonariensis de Frederico Westphalen, RS (quadrado vazado), de União da Vitória, PR (quadrado preenchido) e E. patagonicus de Garruchos, RS (círculos). Círculos de 95\% de confiança.

Em muitas espécies de Molossidae ocorre dimorfismo sexual quanto ao tamanho, com machos geralmente maiores do que as fêmeas (BARQuez et al. 1999, Fábian \& Gregorin 2007). EGER (1977) encontrou diferenças significativas entre sexos em 26\% dos 38 caracteres examinados em E. patagonicus, e Célia López-González (1998, com. pess.) relata fêmeas maiores que machos na amostra examinada de 426 E. patagonicus do Paraguai. O número reduzido de espécimes sul brasileiros disponíveis torna leviana qualquer afirmação sobre presença de dimorfismo sexual, e como as médias de machos e fêmeas para as medidas obtidas são na maioria idênticas, é prudente aguardar um incremento no número de espécimes em coleções para uma análise desta natureza.

\section{Implicações nomenclaturais}

BAUD (1981) considera que as três subespécies, "bonariensisbeckeri-delticus", são de difícil separação e apresentam um continuum de medidas nas amostras paraguaias de pequenos Eumops. Eger (1977) também encontrou espécimes de tamanho intermediário entre $E$. b. bonariensis e $E$. $b$. beckeri.

Neste trabalho aceitamos a validade de E. patagonicus como proposto por BARQUEz et al. (1999) . Desta forma, corroboramos o apresentado por González (2003) atribuindo os espécimes de Garruchos a este táxon. Assim sendo, o espécime de Frederico Westphalen e os espécimes procedentes de União da Vitória devem, à luz das informações disponíveis, serem tratados como E. bonariensis.

\section{Distribuição geográfica}

Os dados aqui apresentados alteram substancialmente o cenário distribucional de E. bonariensis e E. patagonicus no sul do Brasil (Figs 1-4). No estado do Rio Grande do Sul E. bonariensis é agora conhecido de apenas duas localidades: Quinta, no sul do estado (EGER 1977) e Frederico Westphalen no extremo norte do estado. Eumops patagonicus permanece conhecida apenas para Garruchos no extremo noroeste do Rio Grande do Sul, e tem um incremento de três espécimes à série apresentada por GonzÁLEz (2003). Garruchos permanece sendo a única localida- de com registros de E. patagonicus em todo o território brasileiro. No Paraná, E. bonariensis possui registro apenas para União da Vitória no sul do estado, já que os espécimes citados para Douradina e Curitiba não foram localizados na coleção.

\section{Considerações finais}

BARQuez et al. (1999) encontraram em coleções alguns espécimes de E. bonariensis e E. patagonicus identificados como Tadarida brasiliensis. Uma análise mais cuidadosa dos espécimes atribuídos a T. brasiliensis em coleções brasileiras poderá revelar novos espécimes das espécies aqui estudadas.

É provável que com futuros esforços amostrais as distribuições de E. bonariensis e E. patagonicus no sul do Brasil se mostrem mais amplas, e que o aumento de espécimes disponíveis em coleções permita a utilização de ferramentas sistemáticas mais potentes para o esclarecimento do status taxonômico destas formas.

\section{AGRADECIMENTOS}

Teresa C.C. Margarido e Sebastião C. Pereira (MHNCI) e Márcia Jardim (MCN/FZB) pelo acesso às coleções. Célia LópezGonzález, Burton Lim e Fábio A.F. Jacomassa pelo envio de bibliografias. Marcio Pie pela elaboração do Abstract.

\section{LITERATURA CITADA}

Acosta y Lara, E.F. 1950. Quirópteros del Uruguay. Comunicaciones Zoologicas del Museo de Historia Natural de Motevideo 58 (3): 1-73.

BARQUEZ, R.M. 2006. Orden Chiroptera, p. 56-86. In: R.M. BARQUEZ: M.M. Díaz \& R.A. Ojeda (Eds). Mamíferos de Argentina, Sistemática y Distribución. Tucumán, SAREM, 359p.

Barquez, R.M.; M.A. Mares \& J.K. Braun. 1999. The Bats of Argentina. Special Publications Museum of Texas Tech University 42: 1-275.

BAUd, F.J. 1981. Expédition du Muséum de Geneve au Paraguay: Chiroptères. Revue Suisse de Zoologie 88: 567-581.

Cabrera, A. 1957. Catalogo de los mamíferos de América del Sur. Revista del Museo Argentino de Ciencias Naturales "Bernardino Rivadavia", Buenos Aires 4 (1): 1-307.

Dolan, P. 1989. Systematics of Middle American mastiff bats of the genus Molossus. Special Publications Museum of Texas Tech University 29: 1-71.

Eger, J.L. 1977. Systematics of the genus Eumops (Chiroptera: Molossidae). Life Science Contributions, Royal Ontario Museum 110: 1-69.

Fabian, M.E. \& R. Gregorin. 2007. Família Molossidae, p. 149-165. In: N.R. Reis: A.L. Peracchi: W.A. Pedro \& I.P. Lima (Eds). Morcegos do Brasil. Londrina, N.R. Reis, 253p.

Freeman, P.W. 1981. A multivariate study of the family Molossidae (Mammalia: Molossidae): morfology, ecology, evolution. Fieldiana Zoology 7: 1-173.

GonZÁlez, J.C. 2003. Primeiro registro de Eumops patagonicus Thomas, 1924 para o Brasil (Mammalia: Chiroptera: Molossidae). Comunicações do Museu de Ciência e Tecnologia da PUCRS 16 (2): 255-258. 
Gregorin, R. \& V.A. Taddei. 2002. Chave artificial para a identificação de Molossídeos brasileiros (Mammalia, Chiroptera). Mastozoología Neotropical 9 (1): 13-32.

Hunt, J.L.; L.A. McWilliams; T.L. Best \& K.G. Smith. 2003. Eumops bonariensis. Mammalian Species 733: 1-5.

Koopman, K.F. 1994. Order Chiroptera, p. 137-241. In: D.E. WILSON \& D. ReEDER (Eds). Mammal species of the world, a taxonomic and geographic reference. Washington, Smithsonian Institution Press, 2142p.

Mares, M.A.; R.M. Bárquez; J.K. Braun \& R.A. OJeda. 1996. Observations on the mammals of Tucumán Province, Argentina. I. Systematics, distribution, and ecology of the Didelphimorphia, Xenarthra, Chiroptera, Primates, Carnivora, Perissodactyla, Artiodactyla, and Lagomorpha. Annals of Carnegie Museum 65: 89-152.

Miretzki, M. 2003. Morcegos do Estado do Paraná, Brasil (Mammalia, Chiroptera): riqueza de espécies, distribuição e síntese do conhecimento atual. Papéis Avulsos de Zoologia 43 (6): 101-138.

Submitted: 13.VI.2008; Accepted: 13.III.2009.

Editorial responsibility: Ana Lúcia da Costa Prudente
Pacheco, S.M. \& T.R.O. Freitas. 2003. Quirópteros, p. 483-497. In: C.S. Fontana; G.A. Bencke \& R.E. Reis (Eds). Livro vermelho da fauna ameaçada de extinção no Rio Grande do Sul. Porto Alegre, EDIPUCRS, 632p.

Peracchi, A.L.; Lima, I.P.; Reis, N.R.; Nogueira, M.R. \& Filho, H.O. 2006. Ordem Chiroptera, p. 153-230. In: N.R Reis; A.L. Peracchi; W.A. Pedro \& I.P. Lima (Eds). Mamíferos do Brasil. Londrina, $437 \mathrm{p}$.

Sanborn, C.C. 1932. The bats of the genus Eumops. Journal of Mammalogy 13: 347-357.

SILVA, F. 1985. Guia para determinação de morcegos: Rio Grande do Sul. Porto Alegre, Martins Livreiro, 77p.

SILVA, F. 1994. Mamíferos silvestres: Rio Grande do Sul. Porto Alegre, Publicações Avulsas da Fundação Zoobotânica do Rio Grande do Sul, 246p.

Simmons, N.B. 2005. Order Chiroptera, p. 312-529. In: D.E. Wilson \& D. M. Reeder (Eds). Mammals Species of the World: a taxonomic and geographic reference. Baltimore, Johns Hopkins University Press, Baltimore, vol. 1, 2142p. 